Bundesgesundheitsbl 2015 · 58:1349-1350

DOI 10.1007/s00103-015-2250-2

Online publiziert: 20. Oktober 2015

c) Springer-Verlag Berlin Heidelberg 2015

CrossMark

Mitteilungen des Arbeitskreises Blut des Bundesministeriums für Gesundheit

\title{
Feststellung der Spendereignung und Spendetauglichkeit von Hämochromatose Merkmalsträgern
}

\section{Bei der 80. Sitzung des Arbeits- kreises Blut am 31.05.2015 wur- de folgendes Votum (V 45) verab- schiedet:}

\section{Erläuterung zur \\ Hämochromatose (HC) und Darstellung der Situation in der Blutspende}

Gemäß den Richtlinien zur Gewinnung von Blut und Blutbestandteilen und zur Anwendung von Blutprodukten (Hämotherapie) sind Personen, die an chronischen Krankheiten leiden, bei denen die Blutspende eine Gefährdung des Spenders oder des Empfängers nach sich ziehen könnte, auf Dauer von der Spende auszuschließen. Es besteht kein Zweifel, dass die Hämochromatose eine chronische Erkrankung ist, die unbehandelt zu schwerwiegenden Organschädigungen bis zum Tod führen kann. Das Besondere an dieser Erkrankung ist, dass der Aderlass derzeit die Therapie der Wahl darstellt. Werden HC Merkmalsträger rechtzeitig auf diese Weise therapiert, können Folgeschäden der Eisenüberladung vermieden werden, der Ferritinspiegel bleibt im Normbereich und es gibt keine Hinweise darauf, dass Erythrozyten oder Thrombozyten sich in gesunden Merkmalsträgern anders verhalten als die von Personen ohne den Gendefekt. Es bietet sich demnach offensichtlich an, die Blutspende als Therapie für HC Merkmalsträger oder HC Patienten zu nutzen.

Bislang fehlen in Deutschland einheitliche Regeln für den Umgang mit HC

Für den Arbeitskreis Blut

Dr. R. Offergeld, Vorsitzende
Merkmalsträgern als Spender. Auch international ist die Situation sehr heterogen, von einer Zulassung ohne Vorbedingung bis zur Ablehnung von HC Merkmalsträgern als Spender gibt es eine Reihe von Strategien, die sich zwischen diesen Extremen bewegen (Pauwels et al. Vox Sang 2013, 105:121-128).

\section{Definition der HC im Hinblick auf die Spendereignung}

Erworbene HC wurden nicht in die Überlegungen einbezogen, da hier in der Regel andere Erkrankungen zugrunde liegen, die gegen eine Eignung als Spender sprechen.

Die hereditäre HC beruht in ca. 90\% der Fälle auf einer HFE-Mutation, die bis auf den seltenen Typ 4 autosomal rezessiv vererbt wird. In Mitteleuropa sind 5-10 \% der Bevölkerung für das Merkmal heterozygot, 0,2-1\% homozygot. Etwa $80 \%$ der HC Patienten sind homozygot für die C282Y Mutation, etwa 5\% heterozygot für die kombinierte C282Y/H63D Mutation.

Heterozygote zeigen praktisch keine, aber auch Homozygote nur selten Auffälligkeiten sehr unterschiedlicher Ausprägung hinsichtlich Klinik oder Laborparametern. So entwickeln 38-50\% der Homozygoten eine moderate Eisenüberladung mit fraglicher Indikation zum Aderlass und nur 10-33\% entwickeln eine HC-assoziierte Erkrankung mit eindeutiger Indikation zum Aderlass. Ziel der Aderlass-Therapie ist die Aufrechterhaltung eines Serumferritinspiegels von 50-100 $\mu \mathrm{g} / \mathrm{l}$ (EASL clinical guidelines for HFE hemochromatosis in J Hepatol 2010, 53:3-22).
Damit wird deutlich, dass der Genotyp nicht Kriterium für eine Spenderrückstellung sein kann. Stattdessen wird empfohlen, nur Personen mit Eisenüberladung und Folgeschäden der Eisenüberladung an Organen und Geweben als chronisch Erkrankte im Sinne der HämotherapieRichtlinien anzusehen, die dauerhaft von der Spende auszuschließen sind.

3 Kriterien zur Feststellung der Spendereignung und Spendetauglichkeit von HC Merkmalsträgern

Als Spender geeignet sind

a. Asymptomatische homozygote und kombiniert heterozygote HC Merkmalsträger ohne Indikation zum Aderlass. Das entspricht der derzeit akzeptierten Praxis, da viele unerkannte HC Merkmalsträger bereits Spender sind.

b. Asymptomatische homozygote und kombiniert heterozygote HC Merkmalsträger nach erfolgter AderlassTherapie. Alle bekannten Merkmalsträger ohne HC-assoziierte Erkrankung in der Anamnese, die durch regelmäßige Blutspenden ihren Eisenstoffwechsel in der Balance halten und auf diese Weise Serumferritinspiegel im Referenzbereich aufweisen, sind grundsätzlich zur Blutspende geeignet. Der Wert muss innerhalb der letzten 3 Monate vor der Spende bestimmt worden sein. Die Vorgaben der Hämotherapie-Richtlinien hinsichtlich Spendefrequenz und Spendevolumen sind einzuhalten. 
In den Fällen a) und b) entscheidet im Weiteren die tagesaktuelle Feststellung der Spendetauglichkeit über die Zulassung zur Spende.

\section{Offene Fragen, Risiken, Beschreibung des Forschungsbedarfs}

Es bleibt offen, ob Blutspendeeinrichtungen, die diagnostizierte, asymptomatische HC Träger zur Spende zulassen möchten, die Serumferritinbestimmung selbst durchführen oder einen aktuellen Wert des behandelnden Arztes aus den letzten 3 Monaten vor der Spende in die Dokumentation aufnehmen.

Die Besonderheit der Blutspende als kostenlose Therapie könnte zu einer verminderten Adhärenz der HC Spender an die Spenderauswahlkriterien führen. Einrichtungen, die HC Spender zulassen, müssen dem durch eine sorgfältige adäquate Spenderaufklärung entgegenwirken und insbesondere verstärkt auf die Möglichkeit zum freiwilligen Selbstausschluss hinweisen. Außerdem wäre es wünschenswert, wenn den HC Spendewilligen bei nicht gegebener Spendereignung der therapeutische Aderlass in derselben Einrichtung angeboten werden könnte.

Es besteht seitens der Betroffenen der Wunsch, auch schon während der Phase der Eisendepletion, in der Aderlässe im Abstand von 1-2 Wochen erforderlich sind, therapeutisch Blut spenden zu dürfen. Es gibt jedoch keine Studien oder entsprechende Evidenz aus der Literatur, dass diese Spenden mit deutlich erhöhtem Serumferritin keinen negativen Einfluss auf die Präparatequalität und damit die Sicherheit der Empfänger haben. Hier wird Forschungsbedarf für die Fragen gesehen, welchen Einfluss hohe Ferritinspiegel u. a. auf Morphologie und Funktion der Erythrozyten und Thrombozyten, auf das Nebenwirkungsprofil von therapeutischen Plasmen oder die Immunabwehr der betroffenen Personen haben. 